\title{
REGULARIZATION OF PONTRYAGIN MAXIMUM PRINCIPLE IN OPTIMAL CONTROL OF DISTRIBUTED SYSTEMS ${ }^{1}$
}

\author{
Mikhail I. Sumin \\ Nizhnii Novgorod State University, \\ Nizhnii Novgorod, Russia, m.sumin@mail.ru
}

\begin{abstract}
This article is devoted to studying dual regularization method applied to parametric convex optimal control problem of controlled third boundary-value problem for parabolic equation with boundary control and with equality and inequality pointwise state constraints. This dual regularization method yields the corresponding necessary and sufficient conditions for minimizing sequences, namely, the stable, with respect to perturbation of input data, sequential or, in other words, regularized Lagrange principle in nondifferential form and Pontryagin maximum principle for the original problem. Regardless of the fact that the stability or instability of the original optimal control problem, they stably generate a minimizing approximate solutions in the sense of J. Warga for it. For this reason, we can interpret these regularized Lagrange principle and Pontryagin maximum principle as tools for direct solving unstable optimal control problems and reducing to them unstable inverse problems.
\end{abstract}

Key words: Optimal boundary control, Parabolic equation, Minimizing sequence, Dual regularization, Stability, Lagrange principle, Pontryagin maximum principle

\section{Introduction}

Pontryagin maximum principle is the central result of all optimal control theory, including optimal control for differential equations with partial derivatives. Its statement and proof assume, first of all, that the optimal control problem is considered in an ideal situation, when its input data are known exactly. However, in the vast number of important practical problems of optimal control, as well as numerous problems reducing to optimal control problems, the requirement of exact defining input data is very unnatural, and in many undoubtedly interest cases is simply impracticable. In similar problems, we can not, strictly speaking, take as an approximation to the solution of the initial (unperturbed) problem with the exact input data, a control formally satisfying the maximum principle in the perturbed problem. The reason of such situation lies in the natural instability of optimization problems with respect to perturbation of its input data. As a typical property of optimization problems in general, including constrained ones, instability fully manifests itself in optimal control problems (see., e.g., [10]). As a consequence, the above mentioned instability implies "instability" of the classical optimality conditions, including the conditions in the form of Pontryagin maximum principle. This instability manifests itself in selecting arbitrarily distant "perturbed" optimal elements from their unperturbed counterparts in the case of an arbitrarily small perturbations of the input data. The above applies, in full measure, both to discussed below optimal control problem with pointwise state constraints for linear parabolic equation in divergent form, and to the classical optimality conditions in the form of the Lagrange principle and the Pontryagin maximum principle for this problem.

\footnotetext{
${ }^{1}$ This work was supported by the Russian Foundation for Basic Research (project no. 15-47-02294$\mathrm{r}_{-}$povolzh'e_ - a), by the Ministry of Education and Science of the Russian Federation within the framework of project part of state tasks in 2014-2016 (code no. 1727) and by the grant within the agreement of August 27, 2013 No. 02.B.49.21.0003 between the Ministry of Education and Science of the Russian Federation and Lobachevskii State University of Nizhnii Novgorod.
} 
In this paper we discuss how to overcome the problem of instability of the classical optimality conditions in optimal control problems applying dual regularization method (see., e.g., [11-13]) and simultaneous transition to the concept of minimizing sequence of admissible elements as the main concept of optimization theory. The latter role acts the concept of the minimizing approximate solution in the sense of J. Warga [23]. The main attention in the paper is given to the discussion of the so-called regularized or, in other words, stable, with respect to perturbation of input data, sequential Lagrange principle in the nondifferential form and Pontryagin maximum principle. Regardless of the stability or instability of the original optimal control problem, they stably generate minimizing approximate solutions for it. For this reason, we can interpret the regularized Lagrange principle and Pontryagin maximum principle that are obtained in the article as tools for direct solving unstable optimal control problems and reducing to them unstable inverse problems $[10,14-16]$. Thus, they contribute to a significant expansion of the range of applicability of the theory of optimal control in which a central role belongs to classic constructions of the Lagrange and Hamilton-Pontryagin functions. Finally, we note that discussed in this paper regularized Lagrange principle in the nondifferential form and Pontryagin maximum principle may have another kind, more convenient for applications $[4,9,15]$. Justification of these alternative forms of the regularized Lagrange principle and Pontryagin maximum principle is based on the so-called method of iterative dual regularization $[11,12]$. In this case, they take the form of iterative processes with the corresponding stopping rules when the error of input data is fixed and finite. Here these alternative forms are not considered.

\section{Statement of optimal control problem}

We consider the fixed-time parametric optimal control problem

$$
\begin{gathered}
g_{0}^{\delta}(\pi) \rightarrow \min , \quad \pi \equiv(u, w) \in \mathcal{D} \subset L_{2}\left(Q_{T}\right) \times L_{2}\left(S_{T}\right), \\
g_{1}^{\delta}(\pi)(x, t) \equiv \varphi_{1}^{\delta}(x, t) z^{\delta}[\pi](x, t)=h^{\delta}(x, t)+p(x, t) \quad \text { for a.e. }(x, t) \in Q, \\
g_{2}^{\delta}(\pi)(x, t) \equiv \varphi_{2}^{\delta}\left(x, t, z^{\delta}[\pi](x, t)\right) \leq r(x, t) \quad \text { for a.e. }(x, t) \in Q
\end{gathered}
$$

with equality and inequality pointwise state constraints understood as ones in the Hilbert space $\mathcal{H} \equiv L_{2}(Q)$

$$
\mathcal{D} \equiv\left\{u \in L_{2}\left(Q_{T}\right): u(x, t) \in U \text { for a.e. }(x, t) \in Q_{T}\right\} \times\left\{w \in L_{2}\left(S_{T}\right): w(x, t) \in W \text { for a.e. }(x, t) \in S_{T}\right\} ;
$$

$U, W \subset \mathbb{R}^{1}$ are convex compact sets. In this problem, $p \in \mathcal{H}$ and $r \in \mathcal{H}$ are parameters; $g_{0}^{\delta}$ : $L_{2}\left(Q_{T}\right) \times L_{2}\left(S_{T}\right)$ is a continuous convex functional, $Q \subset \bar{Q}_{\iota, T}$ is a compact set without isolated points with a nonempty interior, $\iota \in(0, T), Q=\operatorname{clint} Q$; and $z^{\delta}[\pi] \in V_{2}^{1,0}\left(Q_{T}\right) \cap C\left(\bar{Q}_{T}\right)$ is a weak solution [6] to the third boundary-value problem $^{2}$

$$
\begin{gathered}
z_{t}-\frac{\partial}{\partial x_{i}}\left(a_{i, j}(x, t) z_{x_{j}}\right)+a^{\delta}(x, t) z+u(x, t)=0 \\
z(x, 0)=v_{0}(x), \quad x \in \Omega, \quad \frac{\partial z}{\partial \mathcal{N}}+\sigma^{\delta}(x, t) z=w(x, t), \quad(x, t) \in S_{T} .
\end{gathered}
$$

The superscript $\delta$ in the input data of Problem $\left(P_{p, r}^{\delta}\right)$ indicates that these data are exact $(\delta=0)$ or perturbed $(\delta>0)$, i.e., they are specified with an error, $\delta \in\left[0, \delta_{0}\right]$, where $\delta_{0}>0$ is a fixed number.

\footnotetext{
${ }^{2}$ Here and below, we use the notations for the sets $Q_{T}, S_{T}, Q_{i, T}$ and also for functional spaces and norms of their elements adopted in monograph [6].
} 
For definiteness, as a target functional we take the terminal one

$$
g_{0}^{\delta}(\pi) \equiv \int_{\Omega} G^{\delta}\left(x, z^{\delta}[\pi](x, T)\right) d x .
$$

The input data for Problem $\left(P_{p, r}^{0}\right)$ are assumed to meet the following conditions:

a) It is true that $a_{i, j} \in L_{\infty}\left(Q_{T}\right), i, j=1, \ldots, n, a^{\delta} \in L_{\infty}\left(Q_{T}\right), \sigma^{\delta} \in L_{\infty}\left(S_{T}\right), v_{0}^{\delta} \in C(\bar{\Omega})$,

$$
\begin{gathered}
\nu|\xi|^{2} \leq a_{i, j}(x, t) \xi_{i} \xi_{j} \leq \mu|\xi|^{2} \quad \forall(x, t) \in Q_{T}, \quad \nu, \mu>0, \\
a^{\delta}(x, t) \geq C_{0} \text { for a.e. }(x, t) \in Q_{T}, \quad \sigma^{\delta}(x, t) \geq C_{0} \quad \text { for a.e. }(x, t) \in S_{T} ;
\end{gathered}
$$

b) It is true that $\phi_{1}^{\delta}, h^{\delta} \in L_{\infty}(Q) ; \phi_{2}^{\delta}: Q \times \mathbb{R}^{1} \rightarrow \mathbb{R}^{1}$ is Lebesgue measurable function that is continuous and convex with respect to $z$ for a.e. $(x, t) \in Q, \varphi_{2}^{\delta}(\cdot, \cdot, z(\cdot, \cdot)) \in L_{\infty}(Q)$ $\forall z \in C(Q) ; G^{\delta}: \Omega \times \mathbb{R}^{1} \rightarrow \mathbb{R}^{1}$ is Lebesgue measurable function that is continuous and convex with respect to $z$ for a.e. $x \in \Omega, G^{\delta}(\cdot, z(\cdot, T)) \in L_{\infty}(\Omega) \forall z(\cdot, T) \in C(Q)$;

c) $\Omega \subset \mathbb{R}^{n}$ be a bounded domain with piece-wise smooth boundary $S$.

Assume that the following estimates hold:

$$
\begin{gathered}
\left|G^{\delta}(x, z)-G^{0}(x, z)\right| \leq C_{M} \delta \quad \forall(x, z) \in \Omega \times S_{M}^{1}, \\
\left\|\varphi_{1}^{\delta}-\varphi_{1}^{0}\right\|_{\infty, Q} \leq C \delta, \quad\left\|h^{\delta}-h^{0}\right\|_{\infty, Q} \leq C \delta, \\
\left|\varphi_{2}^{\delta}(x, t, z)-\varphi_{2}^{0}(x, t, z)\right| \leq C_{M} \delta \quad \forall(x, t, z) \in Q \times S_{M}^{1}, \\
\left\|a^{\delta}-a^{0}\right\|_{\infty, Q_{T}} \leq C \delta, \quad\left|v_{0}^{\delta}-v_{0}^{0}\right| \frac{(0)}{\Omega} \leq C \delta, \quad\left\|\sigma^{\delta}-\sigma^{0}\right\|_{\infty, S_{T}} \leq C \delta,
\end{gathered}
$$

where $C, C_{M}>0$ are independent of $\delta ; S_{M}^{n} \equiv\left\{x \in \mathbb{R}^{n}:|x|<M\right\}$. Let's note, that the conditions on the input data of Problem $\left(P_{p, r}^{\delta}\right)$, and also the estimates of deviations of the perturbed input data from the exact ones can be weakened.

\section{Basic concepts and auxiliary propositions}

In this paper we use for discussing the main results, related to the stable sequential Lagrange principle and Pontryagin maximum principle in Problem $\left(P_{p, r}^{0}\right)$, a scheme of studying the similar optimization problems in the papers $[17,19]$ for a system of controlled ordinary differential equations (see also $[20,21]$ for the case of distributed systems). In these works, both spaces of admissible controls and spaces, containing lie images of the operators that define the pointwise state constraints, were presented as Hilbert spaces of square-integrable functions. For this reason, we put the set $\mathcal{D}$ of admissible controls $\pi$ into a Hilbert space also, i.e., assume that

$$
\mathcal{D} \subset Z \equiv L_{2}\left(Q_{T}\right) \times L_{2}\left(S_{T}\right), \quad\|\pi\| \equiv\left(\|u\|_{2, Q_{T}}^{2}+\|w\|_{2, S_{T}}^{2}\right)^{1 / 2} .
$$

At the same time, we note that the conditions on the input data of Problem $\left(P_{p, r}^{\delta}\right)$ allow formally to consider that the operators $g_{1}^{\delta}, g_{2}^{\delta}$, specifying the state constraints of the problem, act into space $L_{p}(Q)$ with any index $p \in[1,+\infty]$. However, in this paper, taking into account the above remark, we will put images of these functional operators in the Hilbert space $L_{2}(Q) \equiv \mathcal{H}$. We note here that the imbedding the images of the operators $g_{1}^{\delta}, g_{2}^{\delta}$, specifying the state constraints, into reflexive space $L_{p}(Q)$ with $1<p<2$, in general, permits significantly to weaken the conditions on the input data and to get, strictly speaking, a stronger result in Problem $\left(P_{p, r}^{0}\right)$. 
If Problem $\left(P_{p, r}^{0}\right)$ is solvable (it has a unique solution if $g_{0}^{0}$ is strictly (strongly) convex), then its solutions are denoted by $\pi_{p, r}^{0} \equiv\left(u_{p, r}^{0}, w_{p, r}^{0}\right)$, and the set of all such solutions is designated as $U_{p, r}^{0}$. Define the Lagrange functional, a set of its minimizers and the concave dual problem

$$
\begin{gathered}
L_{p, r}^{\delta}(\pi, \lambda, \mu) \equiv g_{0}^{\delta}(\pi)+\left\langle\lambda, g_{1}^{\delta}(\pi)-h^{\delta}-p\right\rangle+\left\langle\mu, g_{2}^{\delta}(\pi)-r\right\rangle, \quad \pi \in \mathcal{D}, \\
U^{\delta}[\lambda, \mu] \equiv \operatorname{Argmin}\left\{L_{p, r}^{\delta}(\pi, \lambda, \mu): \pi \in \mathcal{D}\right\} \quad \forall(\lambda, \mu) \in \mathcal{H} \times \mathcal{H}_{+}, \\
\mathcal{H}_{+} \equiv\{z \in \mathcal{H}: z(x, t) \geq 0 \text { for a.e. } \quad(x, t) \in Q\}, \\
V_{p, r}^{\delta}(\lambda, \mu) \rightarrow \sup , \quad(\lambda, \mu) \in \mathcal{H} \times \mathcal{H}_{+}, \quad V_{p, r}^{\delta}(\lambda, \mu) \equiv \inf _{\pi \in \mathcal{D}} L_{p, r}^{\delta}(\pi, \lambda, \mu) .
\end{gathered}
$$

Since the Lagrange functional is continuous and convex for any pair $(\lambda, \mu) \in \mathcal{H} \times \mathcal{H}_{+}$, and the set $\mathcal{D}$ is bounded, the dual functional $V_{p, r}^{\delta}$, is obviously defined and finite for any $(\lambda, \mu) \in \mathcal{H} \times \mathcal{H}_{+}$.

The concept of a minimizing approximate solution in the sense of J. Warga [23] is of great importance for the design of a dual regularizing algorithm for Problem $\left(P_{p, r}^{0}\right)$. Recall that a minimizing approximate solution is a sequence $\pi^{i} \equiv\left(u^{i}, w^{i}\right), i=1,2, \ldots$ such that $g_{0}^{0}\left(\pi^{i}\right) \leq \beta(p, r)+\delta^{i}$, $\pi^{i} \in \mathcal{D}_{p, r}^{0, \epsilon^{i}}$ for some nonnegative number sequences $\delta^{i}$ and $\epsilon^{i}, i=1,2, \ldots$, that converge to zero. Here, $\beta(p, r)$ is the generalized infimum, i.e., $S$-function:

$$
\begin{gathered}
\beta(p, r) \equiv \lim _{\epsilon \rightarrow+0} \beta_{\epsilon}(p, r), \quad \beta_{\epsilon}(p, r) \equiv \inf _{\pi \in \mathcal{D}_{p, r}^{0, \epsilon}} g_{0}^{0}(\pi), \quad \beta_{\epsilon}(p, r) \equiv+\infty \text { if } \mathcal{D}_{p, r}^{0, \epsilon}=\emptyset, \\
\mathcal{D}_{p, r}^{\delta, \epsilon} \equiv\left\{\pi \in \mathcal{D}:\left\|g_{1}^{\delta}(\pi)-h^{\delta}-p\right\|_{2, Q} \leq \epsilon, \min _{z \in \mathcal{H}}\left\|g_{2}^{\delta}(\pi)-r-z\right\|_{2, Q} \leq \epsilon\right\}, \quad \epsilon \geq 0, \\
\mathcal{D}_{p, r}^{00} \equiv \mathcal{D}_{p, r}^{0}, \quad \mathcal{H}_{-} \equiv\{z \in \mathcal{H}: z(x, t) \leq 0 \text { for a.e. }(x, t) \in Q\}, \quad \mathcal{H}_{+} \equiv-\mathcal{H}_{-} .
\end{gathered}
$$

Obviously, in the general situation, $\beta(p, r) \leq \beta_{0}(p, r)$, where $\beta_{0}(p, r)$ is the classical value of the problem. However, in the case of Problem $\left(P_{p, r}^{0}\right)$, we have $\beta(p, r)=\beta_{0}(p, r)$. Simultaneously, we may asset that $\beta: \mathcal{H} \times \mathcal{H} \rightarrow \mathbb{R}^{1} \cup\{+\infty\}$ is a convex and lower semicontinuous function. Note here that the existence of a minimizing approximate solution in Problem $\left(P_{p, r}^{0}\right)$ obviously implies its solvability.

From the conditions a) - c) and from the theorem on the existence of a weak solution of the third boundary-value problem for a linear parabolic equation of the divergent type [6, ch. III, section 5] (see also $[5,7]$ ), it follows that the direct boundary-value problem (1.1) and the corresponding adjoint problem are uniquely solvable in $V_{2}^{1,0}\left(Q_{T}\right)$.

Proposition 1. For any pair $(u, w) \in L_{2}\left(Q_{T}\right) \times L_{2}\left(S_{T}\right)$ and for any $T>0$ the direct boundaryvalue problem (1.1) is uniquely solvable in $V_{2}^{1,0}\left(Q_{T}\right)$ and we have the estimate

$$
\left|z^{\delta}[\pi]\right|_{Q_{T}}+\left\|z^{\delta}[\pi]\right\|_{2, S_{T}} \leq C_{T}\left(\|u\|_{2, Q_{T}}+\left\|v_{0}\right\|_{2, \Omega}+\|w\|_{2, S_{T}}\right)
$$

where the constant $C_{T}$ is independent of $\delta \geq 0$ and pair $\pi \equiv(u, w) \in L_{2}\left(Q_{T}\right) \times L_{2}\left(S_{T}\right)$. Also the adjoint problem

$$
\begin{aligned}
-\eta_{t}-\frac{\partial}{\partial x_{j}} a_{i, j}(x, t) \eta_{x_{i}}+a^{\delta}(x, t) \eta & =\chi(x, t), \\
\eta(x, T)=\psi(x), \quad x \in \Omega, \quad \frac{\partial \eta}{\partial \mathcal{N}}+\sigma^{\delta}(x, t) \eta & =\omega(x, t), \quad(x, t) \in S_{T}
\end{aligned}
$$

is uniquely solvable in $V_{2}^{1,0}\left(Q_{T}\right)$ for any $\chi \in L_{2}\left(Q_{T}\right), \psi \in L_{2}(\Omega), \omega \in L_{2}\left(S_{T}\right)$ and any $T>0$. Its solution is denoted as $\eta[\chi, \psi, \omega]$. Simultaneously, the estimate

$$
\left|\eta^{\delta}[\chi, \psi, \omega]\right|_{Q_{T}}+\left\|\eta^{\delta}[\chi, \psi, \omega]\right\|_{2, S_{T}} \leq C_{T}^{1}\left(\|\chi\|_{2, Q_{T}}+\|\psi\|_{2, \Omega}+\|\omega\|_{2, S_{T}}\right),
$$

is true where the constant $C_{T}^{1}$ is independent of $\delta \geq 0$ and a triple $(\chi, \psi, \omega)$. 
Simultaneously, from conditions a) - c) and the theorems on the existence of a weak (generalized) solution of the third boundary-value problem for a linear parabolic equation of the divergent type (see, e.g., $[3,8]$ ), it follows that the direct boundary-value problem is uniquely solvable in $V_{2}^{1,0}\left(Q_{T}\right) \cap C\left(\bar{Q}_{T}\right)$.

Proposition 2. Let us $l>n+1$. For any pair $(u, w) \in L_{l}\left(Q_{T}\right) \times L_{l}\left(S_{T}\right)$ and any $T>0$, $\delta \in\left[0, \delta_{0}\right]$ the direct boundary-value problem $(1.1)$ is uniquely solvable in $V_{2}^{1,0}\left(Q_{T}\right) \cap C\left(\bar{Q}_{T}\right)$ and the estimate

$$
\left|z^{\delta}[\pi]\right|_{\bar{Q}_{T}}^{(0)} \leq C_{T}\left(\|u\|_{l, Q_{T}}+\left|v_{0}\right|_{\bar{\Omega}}^{(0)}+\|w\|_{l, S_{T}}\right),
$$

is true where the constant $C_{T}$ is independent of pair $\pi \equiv(u, w)$ and $\delta$.

Further, the minimization problem for Lagrange functional

$$
L_{p, r}^{\delta}(\pi, \lambda, \mu) \rightarrow \min , \quad \pi \in \mathcal{D}, \quad \text { when } \quad(\lambda, \mu) \in \mathcal{H} \times \mathcal{H}_{+}
$$

plays the central role in all subsequent constructions. It is usual problem without equality and inequality constraints. It is solvable as a minimization problem for weakly semicontinuous functional on the weak compact set $\mathcal{D} \subset L_{2}\left(Q_{T}\right) \times L_{2}\left(S_{T}\right)$. Here, the weak semicontinuity is a consequence of the convexity and continuity with respect to $\pi$ of the Lagrange functional. Minimizers $\pi^{\delta}[\lambda, \mu] \in U^{\delta}[\lambda, \mu]$ for this optimal control problem satisfy the Pontryagin maximum principle under supplementary assumption of the existence of Lebesgue measurable with respect to $(x, t) \in Q$ for all $z \in \mathbb{R}^{1}$ and continuous with respect to $z$ for a.e. $x, t$ gradients $\nabla_{z} \varphi_{2}^{\delta}(x, t, z), \nabla_{z} G^{\delta}(x, z)$ with the estimates

$$
\left|\nabla_{z} \varphi_{2}^{\delta}(x, t, z)\right| \leq C_{M}, \quad\left|\nabla_{z} G^{\delta}(x, z)\right| \leq C_{M}, \quad \forall z \in S_{M}^{1},
$$

where $C_{M}>0$ is independent of $\delta$. The following lemma is true due to the estimates of the propositions 1, 2 and to the so called two-parameter variation [22] of the pair $\pi^{\delta}[\lambda, \mu]$ that is needle-shaped with respect to control $u$ and classical with respect to control $w$.

Lemma 1. Let $H(y, \eta) \equiv-\eta y$ and the additional condition that specified above is fulfilled. Any pair $\pi^{\delta}[\lambda, \mu]=\left(u^{\delta}[\lambda, \mu], w^{\delta}[\lambda, \mu]\right) \in U^{\delta}[\lambda, \mu],(\lambda, \mu) \in \mathcal{H} \times \mathcal{H}_{+}$satisfies the (usual) Pontryagin maximum principle in the problem (2.1): for $\pi=\pi^{\delta}[\lambda, \mu]$ the following maximum relations

$$
\begin{aligned}
& H\left(u(x, t), \eta^{\delta}(x, t)\right)=\max _{u \in U} H\left(u, \eta^{\delta}(x, t)\right) \text { for a.e. } Q_{T}, \\
& H\left(w(s, t), \eta^{\delta}(s, t)\right)=\max _{w \in W} H\left(w, \eta^{\delta}(s, t)\right) \text { for a.e. } S_{T}
\end{aligned}
$$

hold, where $\eta^{\delta}(x, t),(x, t) \in Q_{T}$ is a solution for $\pi=\pi^{\delta}[\lambda, \mu]$ of the adjoint problem

$$
\begin{gathered}
-\eta_{t}-\frac{\partial}{\partial x_{j}}\left(a_{i, j}(x, t) \eta_{x_{i}}\right)+a^{\delta}(x, t) \eta=\varphi_{1}^{\delta}(x, t) \lambda(x, t)+\nabla_{z} \varphi_{2}^{\delta}\left(x, t, z^{\delta}[\pi](x, t)\right) \mu(x, t), \quad(x, t) \in Q_{T}, \\
\eta(x, T)=\nabla_{z} G^{\delta}\left(x, z^{\delta}[\pi](x, T)\right), \quad x \in \Omega \\
\frac{\partial \eta(x, t)}{\partial \mathcal{N}}+\sigma^{\delta}(x, t) \eta=0, \quad(x, t) \in S_{T} .
\end{gathered}
$$

Remark 1. Note that here and below, if the functions $\varphi_{1}^{\delta}, \nabla_{z} \varphi_{2}^{\delta}(\cdot, \cdot, z(\cdot, \cdot)), \lambda, \mu \in \mathcal{H}$ are considered on the entire cylinder $Q_{T}$, we set that the equalities $\varphi_{1}^{\delta}(x, t)=\nabla_{z} \varphi_{2}^{\delta}(x, t, z(x, t))=$ $\lambda(x, t)=\mu(x, t)=0$ take place for $(x, t) \in Q_{T} \backslash Q$; the same notation is preserved if these functions are taken on the entire cylinder.

An important result for the subsequent presentation is the following lemma, which is a consequence of the classical asymmetric minimax theorem [2, Chapter 6, Section 2, Theorem 7]. 
Lemma 2. The minimax equality

$$
\inf _{\pi \in \mathcal{D}} \sup _{(\lambda, \mu) \in \mathcal{H} \times \mathcal{H}_{+}} L_{p, r}^{0}(\pi, \lambda, \mu)=\sup _{(\lambda, \mu) \in \mathcal{H} \times \mathcal{H}_{+}} \inf _{\pi \in \mathcal{D}} L_{p, r}^{0}(\pi, \lambda, \mu)
$$

is true. It can be rewritten as the duality relation

$$
g_{0}^{0}\left(\pi_{p, r}^{0}\right)=\sup _{(\lambda, \mu) \in \mathcal{H} \times \mathcal{H}_{+}} V_{p, r}^{0}(\lambda, \mu) .
$$

In the next section we construct minimizing approximate solutions for Problem $\left(P_{p, r}^{0}\right)$ from the elements $\left.\pi^{\delta}[\lambda, \mu],(\lambda, \mu) \in \mathcal{H}\right) \times \mathcal{H}_{+}$. As consequence, this construction leads us to various versions of the stable sequential Lagrange principle and Pontragin maximum principle. In the case of strong convexity and subdifferentiability of the target functional $g_{0}^{0}$, these versions are statements about stable approximations of the solutions of Problem $\left(P_{p, r}^{0}\right)$ in the metric of $Z \equiv L_{2}\left(Q_{T}\right) \times L_{2}\left(S_{T}\right)$ by the points $\pi^{\delta}[\lambda, \mu]$. Due to the estimates (1.2) and the propositions 1,2 we may assert that the estimates

$$
\begin{gathered}
\left|g_{0}^{\delta}(\pi)-g_{0}^{0}(\pi)\right| \leq C_{1} \delta \quad \forall \pi \in \mathcal{D}, \quad\left\|g_{1}^{\delta}(\pi)-g_{1}^{0}(\pi)\right\|_{2, Q} \leq C_{2} \delta(1+\|\pi\|) \quad \forall \pi \in Z, \\
\left\|h^{\delta}-h^{0}\right\|_{2, Q} \leq C \delta, \quad\left\|g_{2}^{\delta}(\pi)-g_{2}^{0}(\pi)\right\|_{2, Q} \leq C_{3} \delta \quad \forall \pi \in \mathcal{D},
\end{gathered}
$$

hold, in which the constants $C_{1}, C_{2}, C_{3}>0$ are independent of $\delta \in\left(0, \delta_{0}\right], \pi$.

Since the set $\mathcal{D}$ is bounded, the dual functional is obviously defined and finite for any element $(\lambda, \mu) \in \mathcal{H} \times \mathcal{H}$. Moreover, it is also obvious that the value $V_{p, r}^{\delta}(\lambda, \mu)$ is reached at elements $\pi^{\delta}[\lambda, \mu]$ of the set $U^{\delta}[\lambda, \mu] \equiv \operatorname{Argmin}\left\{L_{p, r}^{\delta}(\pi, \lambda, \mu), \pi \in \mathcal{D}\right\}$ for $(\lambda, \mu) \in \mathcal{H} \times \mathcal{H}_{+}$,

$$
\mathcal{H}_{+} \equiv\{z \in \mathcal{H}: z(x, t) \geq 0 \text { for a.e. }(x, t) \in Q\} .
$$

Note also that, by virtue of estimates (2.4) and since $\mathcal{D}$ is bounded, we have the estimate

$$
\left|V_{p, r}^{\delta}(\lambda, \mu)-V_{p, r}^{0}(\lambda, \mu)\right| \leq C \delta(1+\|\lambda\|+\|\mu\|),
$$

where $C>0$ is a constant independent of $\lambda, \mu, \delta$.

\section{Stable sequential Pontryagin maximum principle}

In this section we discuss the so-called regularized or, in other words, stable, with respect to errors of input data, sequential Pontryagin maximum principle for Problem $\left(P_{p, r}^{0}\right)$ as necessary and sufficient condition for elements of minimizing approximate solutions. Simultaneously, we may treat this condition as one for existence of a minimizing approximate solutions in Problem $\left(P_{p, r}^{0}\right)$ with perturbed input data or as condition of stable construction of a minimizing sequence in this problem. The proof of the necessity of this condition is based on the dual regularization method [11-13] that is a stable algorithm of constructing a minimizing approximate solutions in Problem $\left(P_{p, r}^{0}\right)$.

\subsection{Dual regularization for optimal control problem with pointwise state constraints}

The estimates (2.4) give a possibility to organize the procedure of the dual regularization in accordance with a scheme of the paper [19] for constructing a minimizing approximate solution in Problem $\left(P_{p, r}^{0}\right)$. In accordance with this scheme the dual regularization consists in the direct solving dual of Problem $\left(P_{p, r}^{0}\right)$ and Tikhonov stabilized problem

$$
R_{p, r}^{\delta, \alpha(\delta)}(\lambda, \mu) \equiv V_{p, r}^{\delta}(\lambda, \mu)-\alpha(\delta)\|(\lambda, \mu)\|^{2} \rightarrow \max , \quad(\lambda, \mu) \in \mathcal{H} \times \mathcal{H}_{+}
$$


under consistency condition

$$
\frac{\delta}{\alpha(\delta)} \rightarrow 0, \quad \alpha(\delta) \rightarrow 0, \quad \delta \rightarrow 0
$$

Let us denote $\left(\lambda_{p, r}^{\delta, \alpha}, \mu_{p, r}^{\delta, \alpha}\right) \equiv \operatorname{argmax}\left\{R_{p, r}^{\delta, \alpha}(\lambda, \mu):(\lambda, \mu) \in \mathcal{H} \times \mathcal{H}_{+}\right\}$. The above dual regularization leads to constructing minimizing approximate solution in Problem $\left(P_{p, r}^{0}\right)$ from the elements $\pi^{\delta}\left[\lambda_{p, r}^{\delta, \alpha(\delta)}, \mu_{p, r}^{\delta, \alpha(\delta)}\right] \in \operatorname{Argmin}\left\{L_{p, r}^{\delta}(\pi, \lambda, \mu): \pi \in \mathcal{D}\right\}$, when $\delta \rightarrow 0$.

In this section, we extend the algorithm of the dual regularization $[12,18]$ to the case of Problem $\left(P_{p, r}^{0}\right)$ in which the objective functional is only convex. Below we prove convergence theorem for dual regularization method in exact accordance with a scheme of proving the similar theorem in [19]. We note only that, as in [19], this proving uses a weak continuity of the operators $g_{1}^{\delta}, g_{2}^{\delta}$ that is consequence of the conditions on the input data of Problem $\left(P_{p, r}^{0}\right)$ and a regularity of the bounded solutions of the boundary-value problem (1.1) (see Proposition 2) inside of the cylinder $Q_{T}$ [6, ch.III, theorem 10.1].

Let Problem $\left(P_{p, r}^{0}\right)$ be solvable. To prove the convergence theorem for dual regularization method, first of all, we give a formula for the superdifferential (in the sense of a convex analysis) of the concave value functional $V_{p, r}^{\delta}$. The proof of this formula can be found in [12].

Lemma 3. The superdifferential of the concave value functional $V_{p, r}^{\delta}(\lambda, \mu)$ at the point $(\lambda, \mu) \in$ $\mathcal{H} \times \mathcal{H}$ is equal

$$
\begin{gathered}
\partial V_{p, r}^{\delta}(\lambda, \mu)=\partial_{C} V_{p, r}^{\delta}(\lambda, \mu)=\operatorname{cl} \operatorname{conv}\left\{w-\lim _{i \rightarrow \infty}\left(g_{1}^{\delta}\left(u^{i}\right)-h^{\delta}-p, g_{2}^{\delta}\left(u^{i}\right)-r\right): \pi^{i} \in \mathcal{D},\right. \\
\left.L_{p, r}^{\delta}\left(\pi^{i}, \lambda, \mu\right) \rightarrow \inf _{\pi \in \mathcal{D}} L_{p, r}^{\delta}(\pi, \lambda, \mu), i \rightarrow \infty\right\},
\end{gathered}
$$

where $\partial_{C} V_{p, r}^{\delta}(\lambda, \mu)$ is Clarke's generalized gradient of the functional $V_{p, r}^{\delta}(\lambda, \mu)$ at the point $(\lambda, \mu)$ and the limit $w-\lim$ is understood in the sense of weak convergence in the space $\mathcal{H} \times \mathcal{H}$.

Further, to substantiate the dual regularization method in the case under consideration, we write the inequality $\forall\left(\lambda^{\prime}, \mu^{\prime}\right) \in \mathcal{H} \times \mathcal{H}_{+}$

$$
\left\langle\left(I_{1}, I_{2}\right)-2 \alpha(\delta)\left(\lambda_{p, r}^{\delta, \alpha(\delta)}, \mu_{p, r}^{\delta, \alpha(\delta)}\right),\left(\lambda^{\prime}, \mu^{\prime}\right)-\left(\lambda_{p, r}^{\delta, \alpha(\delta)}, \mu_{p, r}^{\delta, \alpha(\delta)}\right)\right\rangle \leq 0
$$

for some element $\left(I_{1}, I_{2}\right) \in \partial V_{p, r}^{\delta}\left(\lambda_{p, r}^{\delta, \alpha(\delta)}, \mu_{p, r}^{\delta, \alpha(\delta)}\right)$.

By Lemma 3 and the classical properties of closed convex hulls (see [1, p. 210, 217]), we obtain

$$
\begin{gathered}
\left\langle\lim _{s \rightarrow \infty} \sum_{i=1}^{l(s, \delta)} \gamma_{i}(s, \delta)\left(w-\lim _{j \rightarrow \infty}\left(g_{1}^{\delta}\left(\pi_{s, i}^{j}\right)-h^{\delta}-p, g_{2}^{\delta}\left(\pi_{s, i}^{j}\right)\right)-r\right)-2 \alpha(\delta)\left(\lambda_{p, r}^{\delta, \alpha(\delta)}, \mu_{p, r}^{\delta, \alpha}(\delta)\right),\right. \\
\left.\left(\lambda^{\prime}, \mu^{\prime}\right)-\left(\lambda_{p, r}^{\delta, \alpha(\delta)}, \mu_{p, r}^{\delta, \alpha(\delta)}\right)\right\rangle \leq 0 \quad \forall\left(\lambda^{\prime}, \mu^{\prime}\right) \in \mathcal{H} \times \mathcal{H}_{+},
\end{gathered}
$$

where $\sum_{i=1}^{l(s, \delta)} \gamma_{i}(s, \delta)=1, \gamma_{i}(s, \delta) \geq 0, i=1, \ldots, l(s, \delta)$, and $\pi_{s, i}^{j} \in \mathcal{D}, j=1,2, \ldots$ is a sequence such that

$$
L_{p, r}^{\delta}\left(\pi_{s, i}^{j}, \lambda_{p, r}^{\delta, \alpha(\delta)}, \mu_{p, r}^{\delta, \alpha(\delta)}\right) \rightarrow \min _{\pi \in \mathcal{D}} L_{p, r}^{\delta}\left(\pi, \lambda_{p, r}^{\delta, \alpha(\delta)}, \mu_{p, r}^{\delta, \alpha(\delta)}\right), j \rightarrow \infty .
$$

Assume without loss of generality that the sequence $\pi_{s, i}^{j} \in \mathcal{D}, j=1,2, \ldots$, converges weakly as $j \rightarrow \infty$ to an element $\pi_{s, i} \in \mathcal{D}$, which obviously belongs to the set $U^{\delta}\left[\lambda_{p, r}^{\delta, \alpha(\delta)}, \mu_{p, r}^{\delta, \alpha(\delta)}\right]$. Due to weak continuity of the operators $g_{i}^{\delta}, i=1,2$, and boundedness of $\mathcal{D}$, from (3.2) the inequality follows

$$
\left\langle\lim _{s \rightarrow \infty} \sum_{i=1}^{l(s, \delta)} \gamma_{i}(s, \delta)\left(g_{1}^{\delta}\left(\pi_{s, i}\right)-h^{\delta}-p, g_{2}^{\delta}\left(\pi_{s, i}\right)-r\right)-2 \alpha(\delta)\left(\lambda_{p, r}^{\delta, \alpha(\delta)}, \mu_{p, r}^{\delta, \alpha(\delta)}\right),\right.
$$




$$
\left.\left(\lambda^{\prime}, \mu^{\prime}\right)-\left(\lambda_{p, r}^{\delta, \alpha(\delta)}, \mu_{p, r}^{\delta, \alpha(\delta)}\right)\right\rangle \leq 0 \quad \forall\left(\lambda^{\prime}, \mu^{\prime}\right) \in \mathcal{H} \times \mathcal{H}_{+} .
$$

The above inequality implies the limit relations

$$
\begin{gathered}
\lim _{s \rightarrow \infty} \sum_{i=1}^{l(s, \delta)} \gamma_{i}(s, \delta)\left(g_{1}^{\delta}\left(\pi_{s, i}\right)-h^{\delta}-p\right)=2 \alpha(\delta) \lambda_{p, r}^{\delta, \alpha(\delta)}, \\
\lim _{s \rightarrow \infty} \sum_{i=1}^{l(s, \delta)} \gamma_{i}(s, \delta)\left(g_{2}^{\delta}\left(\pi_{s, i}\right)(x, t)-r(x, t)\right)=2 \alpha(\delta) \mu_{p, r}^{\delta, \alpha(\delta)}(x, t) \\
\text { for a.e. }(x, t) \in\left\{(x, t) \in Q: \mu_{p, r}^{\delta, \alpha(\delta)}(x, t)>0\right\}, \\
\lim _{s \rightarrow \infty} \sum_{i=1}^{l(s, \delta)} \gamma_{i}(s, \delta)\left(g_{2}^{\delta}\left(\pi_{s, i}\right)(x, t)-r(x, t)\right) \leq 0 \quad \text { for a.e. }(x, t) \in\left\{(x, t) \in Q: \mu_{p, r}^{\delta, \alpha(\delta)}(x, t)=0\right\} .
\end{gathered}
$$

In turn, the limit relations (3.3)-(3.5) imply the limit equalities

$$
\begin{gathered}
\lim _{s \rightarrow \infty}\left\langle\sum_{i=1}^{l(s, \delta)} \gamma_{i}(s, \delta)\left(g_{1}^{\delta}\left(\pi_{s, i}\right)-h^{\delta}-p\right), \lambda_{p, r}^{\delta, \alpha(\delta)}\right\rangle=2 \alpha(\delta)\left\|\lambda_{p, r}^{\delta, \alpha(\delta)}\right\|^{2} \geq 0 \\
\lim _{s \rightarrow \infty}\left\langle\sum_{i=1}^{l(s, \delta)} \gamma_{i}(s, \delta) g_{2}^{\delta}\left(\pi_{s, i}\right)-r, \mu_{p, r}^{\delta, \alpha(\delta)}\right\rangle=2 \alpha(\delta)\left\|\mu_{p, r}^{\delta, \alpha(\delta)}\right\|^{2} \geq 0 .
\end{gathered}
$$

From (3.4) we obtain also: if $\mu_{p, r}^{\delta, \alpha(\delta)}(x, t)>0$ for some $(x, t)$ belonging to a set of full measure in $\left\{(x, t) \in Q: \mu_{p, r}^{\delta, \alpha(\delta)}(x, t)>0\right\}$, then

$$
\begin{gathered}
\lim _{s \rightarrow \infty} \sum_{i=1}^{l(s, \delta)} \gamma_{i}(s, \delta)\left(g_{2}^{\delta}\left(\pi_{s, i}\right)(x, t)-r(x, t)\right)-2 \alpha(\delta) \mu_{p, r}^{\delta, \alpha(\delta)}(x, t)=0 \\
\lim _{s \rightarrow \infty} \sum_{i=1}^{l(s, \delta)} \gamma_{i}(s, \delta)\left(g_{2}^{\delta}\left(\pi_{s, i}\right)(x, t)-r(x, t)\right) \mu_{p, r}^{\delta, \alpha(\delta)}(t)>0
\end{gathered}
$$

This implies that for a.e. $(x, t) \in Q$ such that $\lim _{s \rightarrow \infty} \sum_{i=1}^{l(s, \delta)} \gamma_{i}(s, \delta)\left(g_{2}^{\delta}\left(\pi_{s, i}\right)(x, t)-r(x, t)\right)<0$, the equality $\mu_{p, r}^{\delta, \alpha(\delta)}(x, t)=0$ holds. From (3.4) and (3.7) we obtain simultaneously that

$$
\mu_{p, r}^{\delta, \alpha(\delta)}(x, t) \lim _{s \rightarrow \infty} \sum_{i=1}^{l(s, \delta)} \gamma_{i}(s, \delta)\left(g_{2}^{\delta}\left(\pi_{s, i}\right)(x, t)-r(x, t)\right) \geq 0 \quad \text { for a.e. }(x, t) \in Q .
$$

Besides, from (3.6) we get the inequality

$$
\begin{gathered}
\lim _{s \rightarrow \infty}\left\langle\sum_{i=1}^{l(s, \delta)} \gamma_{i}(s, \delta)\left(g_{1}^{\delta}\left(\pi_{s, i}\right)-h^{\delta}-p, g_{2}^{\delta}\left(\pi_{s, i}\right)-r\right),\left(\lambda_{p, r}^{\delta, \alpha(\delta)}, \mu_{p, r}^{\delta, \alpha(\delta)}\right)\right\rangle= \\
2 \alpha(\delta)\left(\left\|\lambda_{p, r}^{\delta, \alpha(\delta)}\right\|^{2}+\left\|\mu_{p, r}^{\delta, \alpha(\delta)}\right\|^{2}\right) \geq 0 .
\end{gathered}
$$

Further, since for any $\pi_{p, r}^{0} \in U_{p, r}^{0}$

$$
L_{p, r}^{\delta}\left(\pi_{s, i}, \lambda_{p, r}^{\delta, \alpha(\delta)}, \mu_{p, r}^{\delta, \alpha(\delta)}\right) \equiv g_{0}^{\delta}\left(\pi_{s, i}\right)+\left\langle\lambda_{p, r}^{\delta, \alpha(\delta)}, g_{1}^{\delta}\left(\pi_{s, i}\right)-h^{\delta}-p\right\rangle+\left\langle\mu_{p, r}^{\delta, \alpha(\delta)}, g_{2}^{\delta}\left(\pi_{s, i}\right)-r\right\rangle \leq
$$




$$
\begin{gathered}
L_{p, r}^{\delta}\left(\pi_{p, r}^{0}, \lambda_{p, r}^{\delta, \alpha(\delta)}, \mu_{p, r}^{\delta, \alpha(\delta)}\right) \equiv g_{0}^{\delta}\left(\pi_{p, r}^{0}\right)+\left\langle\lambda_{p, r}^{\delta, \alpha(\delta)}, g_{1}^{\delta}\left(\pi_{p, r}^{0}\right)-h^{\delta}-p\right\rangle+\left\langle\mu_{p, r}^{\delta, \alpha(\delta)}, g_{2}^{\delta}\left(\pi_{p, r}^{0}\right)-r\right\rangle \leq \\
g_{0}^{0}\left(\pi_{p, r}^{0}\right)+\left[g_{0}^{\delta}\left(\pi_{p, r}^{0}\right)-g_{0}^{0}\left(\pi_{p, r}^{0}\right)\right]+\left\|\lambda_{p, r}^{\delta, \alpha}(\delta)\right\|\left\|g_{1}^{\delta}\left(\pi_{p, r}^{0}\right)-h^{\delta}-p\right\|+\left\|\mu_{p, r}^{\delta, \alpha}(\delta)\right\|\left\|g_{2}^{\delta}\left(\pi_{p, r}^{0}\right)-g_{2}^{0}\left(\pi_{p, r}^{0}\right)\right\|,
\end{gathered}
$$

due to the estimates (2.4) and the limit equality (3.8) and doing some elementary transformation, we obtain the estimate

$$
\begin{gathered}
2 \alpha(\delta)\left(\left\|\lambda_{p, r}^{\delta, \alpha(\delta)}\right\|^{2}+\left\|\mu_{p, r}^{\delta, \alpha(\delta)}\right\|^{2}\right) \leq \\
C_{1} \delta\left\|\lambda_{p, r}^{\delta, \alpha(\delta)}\right\|+C_{1} \delta\left\|\mu_{p, r}^{\delta, \alpha(\delta)}\right\|+g_{0}^{0}\left(\pi_{p, r}^{0}\right)+C_{1} \delta-\min _{\pi \in \mathcal{D}} g_{0}^{\delta}(\pi) \leq \\
\sqrt{2} C_{1} \delta \sqrt{\left\|\lambda_{p, r}^{\delta, \alpha(\delta)}\right\|^{2}+\left\|\mu_{p, r}^{\delta, \alpha(\delta)}\right\|^{2}}+g_{0}^{0}\left(\pi_{p, r}^{0}\right)+C_{1} \delta-\min _{\pi \in \mathcal{D}} g_{0}^{\delta}(\pi)
\end{gathered}
$$

or

$$
\alpha(\delta)\left(\left\|\lambda_{p, r}^{\delta, \alpha(\delta)}\right\|^{2}+\left\|\mu_{p, r}^{\delta, \alpha(\delta)}\right\|^{2}\right)-C_{2} \delta \sqrt{\left\|\lambda_{p, r}^{\delta, \alpha(\delta)}\right\|^{2}+\left\|\mu_{p, r}^{\delta, \alpha(\delta)}\right\|^{2}}-g_{0}^{0}\left(\pi_{p, r}^{0}\right)-C_{1} \delta+\min _{\pi \in \mathcal{D}} g_{0}^{\delta}(\pi) \leq 0,
$$

where $C_{1}, C_{2}>0$ are independent of constant $\delta$. From here, the estimate follows

$$
\sqrt{\left\|\lambda_{p, r}^{\delta, \alpha(\delta)}\right\|^{2}+\left\|\mu_{p, r}^{\delta, \alpha(\delta)}\right\|^{2}} \leq \frac{C_{2} \delta+\sqrt{\left(C_{2} \delta\right)^{2}-4 \alpha(\delta) K(\delta)}}{2 \alpha(\delta)}
$$

where $K(\delta) \equiv \min _{\pi \in \mathcal{D}} g_{0}^{\delta}(\pi)-g_{0}^{0}\left(\pi_{p, r}^{0}\right)-C \delta$. In turn, this estimate implies the limit realtions

$$
\alpha(\delta)\left\|\lambda_{p, r}^{\delta, \alpha(\delta)}\right\| \rightarrow 0, \quad \alpha(\delta)\left\|\mu_{p, r}^{\delta, \alpha(\delta)}\right\| \rightarrow 0, \quad \delta \rightarrow 0 .
$$

Further, the limit relations (3.3)-(3.5), (3.9) imply

$$
\begin{gathered}
\lim _{s \rightarrow \infty} \sum_{i=1}^{l(s, \delta)} \gamma_{i}(s, \delta)\left(g_{1}^{\delta}\left(\pi_{s, i}\right)-h^{\delta}-p\right) \rightarrow 0, \quad \delta \rightarrow 0, \\
\lim _{s \rightarrow \infty} \sum_{i=1}^{l(s, \delta)} \gamma_{i}(s, \delta)\left(g_{2}^{\delta}\left(\pi_{s, i}\right)-r\right) \leq \phi(\delta),\|\phi(\delta)\| \rightarrow 0, \quad \delta \rightarrow 0,
\end{gathered}
$$

where the inequality $\lim _{s \rightarrow \infty} \sum_{i=1}^{l(s, \delta)} \gamma_{i}(s, \delta)\left(g_{2}^{\delta}\left(\pi_{s, i}\right)-r\right) \leq \phi(\delta)$ is understood in the sense of ordering on the cone of nonpositive functions $\mathcal{H}_{-}$.

Denoting by $\pi_{\delta} \in U^{\delta}\left[\lambda_{p, r}^{\delta, \alpha(\delta)}, \mu_{p, r}^{\delta, \alpha(\delta)}\right]$ any weak limit point of the sequence $\sum_{i=1}^{l(s, \delta)} \gamma_{i}(s, \delta) \pi_{s, i}$, $s=1,2, \ldots$ and taking into account the inequality

$$
g_{2}^{\delta}\left(\sum_{i=1}^{l(s, \delta)} \gamma_{i}(s, \delta) \pi_{s, i}\right) \leq \sum_{i=1}^{l(s, \delta)} \gamma_{i}(s, \delta) g_{2}^{\delta}\left(\pi_{s, i}\right),
$$

which is understood also in the sense of ordering on the cone of nonpositive functions, we obtain the limit relations

$$
g_{1}^{\delta}\left(\pi_{\delta}\right)-h^{\delta}-p \rightarrow 0, \quad g_{2}^{\delta}\left(\pi_{\delta}\right)-r \leq \lim _{s \rightarrow \infty} \sum_{i=1}^{l(s, \delta)} \gamma_{i}(s, \delta)\left(g_{2}^{\delta}\left(\pi_{s, i}\right)-r\right) \leq \phi(\delta), \quad \delta \rightarrow 0,
$$

and, as a consequence, due to the boundedness of $\mathcal{D}$, the limit relations

$$
g_{1}^{0}\left(\pi_{\delta}\right)-h^{0}-p \rightarrow 0, \quad g_{2}^{0}\left(\pi_{\delta}\right)-r \leq \bar{\phi}(\delta), \quad\|\bar{\phi}(\delta)\| \rightarrow 0, \quad \delta \rightarrow 0 .
$$


Simultaneously, due to the inclusion $\pi_{s, i} \in U^{\delta}\left[\lambda_{p, r}^{\delta, \alpha(\delta)}, \mu_{p, r}^{\delta, \alpha(\delta)}\right]$ we have the inequality

$$
\begin{gathered}
g_{0}^{\delta}\left(\pi_{s, i}\right)+\left\langle\lambda_{p, r}^{\delta, \alpha(\delta)}, g_{1}^{\delta}\left(\pi_{s, i}\right)-h^{\delta}-p\right\rangle+\left\langle\mu_{p, r}^{\delta, \alpha(\delta)}, g_{2}^{\delta}\left(\pi_{s, i}\right)-r\right\rangle \leq \\
g_{0}^{\delta}(\pi)+\left\langle\lambda_{p, r}^{\delta, \alpha(\delta)}, g_{1}^{\delta}(\pi)-h^{\delta}-p\right\rangle+\left\langle\mu_{p, r}^{\delta, \alpha(\delta)}, g_{2}^{\delta}(\pi)-r\right\rangle \quad \forall \pi \in \mathcal{D} .
\end{gathered}
$$

Hence, due to the limit relation (3.8), we can write for any $u_{p, r}^{0} \in U_{p, r}^{0}$

$$
\liminf _{s \rightarrow \infty} \sum_{i=1}^{l(s, \delta)} \gamma_{i}(s, \delta) g_{0}^{\delta}\left(\pi_{s, i}\right) \leq g_{0}^{\delta}\left(\pi_{p, r}^{0}\right)+\left\langle\lambda_{p, r}^{\delta, \alpha(\delta)}, g_{1}^{\delta}\left(\pi_{p, r}^{0}\right)-h^{\delta}-p\right\rangle+\left\langle\mu_{p, r}^{\delta, \alpha(\delta)}, g_{2}^{\delta}\left(\pi_{p, r}^{0}\right)-r\right\rangle
$$

In turn, from here, due to the consistency condition (3.1), the estimates (1.2) and the boundedness of $\mathcal{D}$ we derive

$$
\liminf _{s \rightarrow \infty} \sum_{i=1}^{l(s, \delta)} \gamma_{i}(s, \delta) g_{0}^{0}\left(\pi_{s, i}\right) \leq g_{0}^{0}\left(\pi_{p, r}^{0}\right)+\tilde{\phi}(\delta), \quad \tilde{\phi}(\delta) \rightarrow 0, \quad \delta \rightarrow 0
$$

or

$$
\begin{gathered}
g_{0}^{0}\left(\pi_{\delta}\right) \leq \liminf _{s \rightarrow \infty} g_{0}^{0}\left(\sum_{i=1}^{l(s, \delta)} \gamma_{i}(s, \delta) \pi_{s, i}\right) \leq \liminf _{s \rightarrow \infty} \sum_{i=1}^{l(s, \delta)} \gamma_{i}(s, \delta) g_{0}^{0}\left(\pi_{s, i}\right) \leq \\
g_{0}^{0}\left(\pi_{p, r}^{0}\right)+\tilde{\phi}(\delta), \quad \tilde{\phi}(\delta) \rightarrow 0, \quad \delta \rightarrow 0 .
\end{gathered}
$$

Thus, by virtue of the boundedness of $\mathcal{D}$, weak lower semicontinuity of $g_{0}^{0}$ and weak continuity of $g_{i}^{0}, i=1,2$, we constructed the family of elements $\pi_{\delta} \in U^{\delta}\left[\lambda_{p, r}^{\delta, \alpha(\delta)}, \mu_{p, r}^{\delta, \alpha(\delta)}\right]$, depending on $\delta$, such that the limit relations (3.10) hold and simultaneously

$$
g_{0}^{0}\left(\pi_{\delta}\right) \rightarrow \min _{\pi \in \mathcal{D}_{p, r}^{0}} g_{0}^{0}(\pi), \quad \delta \rightarrow 0 .
$$

Moreover, weak limit point $\bar{\pi}$ of any weakly converging sequence $\pi_{\delta^{k}}, k=1,2, \ldots, \delta^{k} \rightarrow 0, k \rightarrow \infty$, is obviously a solution of Problem $\left(P_{p, r}^{0}\right)$.

We can assert that simultaneously the family of elements $\left(\lambda_{p, r}^{\delta, \alpha(\delta)}, \mu_{p, r}^{\delta, \alpha(\delta)}\right)$, in view of the estimates (1.2), (2.5) and the consistency condition (3.1), satisfies the limit relation (see $[12,13,15,18])$

$$
\lim _{\delta \rightarrow+0} V_{p, r}^{0}\left(\lambda_{p, r}^{\delta, \alpha(\delta)}, \mu_{p, r}^{\delta, \alpha(\delta)}\right)=\sup _{(\lambda, \mu) \in \mathcal{H} \times \mathcal{H}_{+}} V_{p, r}^{0}(\lambda, \mu),
$$

which, combined with the estimate (2.5), the consistency condition (3.1), and the duality relation (2.3) yields the limit relation (see $[12,13,15,18]$ )

$$
\left\langle\left(\lambda_{p, r}^{\delta, \alpha(\delta)}, \mu_{p, r}^{\delta, \alpha(\delta)}\right),\left(g_{1}^{\delta}\left(\pi_{\delta}\right)-h^{\delta}-p, g_{2}^{\delta}\left(\pi_{\delta}\right)\right)-r\right\rangle \rightarrow 0, \quad \delta \rightarrow 0 .
$$

Let us prove the limit relation (3.11). Since

$$
\begin{aligned}
& V_{p, r}^{\delta}\left(\lambda_{p, r}^{\delta, \alpha(\delta)}, \mu_{p, r}^{\delta, \alpha(\delta)}\right)-\alpha(\delta)\left\|\lambda_{p, r}^{\delta, \alpha(\delta)}\right\|^{2}-\alpha(\delta)\left\|\mu_{p, r}^{\delta, \alpha(\delta)}\right\|^{2} \geq \\
& V_{p, r}^{\delta}(\lambda, \mu)-\alpha(\delta)\|\lambda\|^{2}-\alpha(\delta)\|\mu\|^{2} \quad \forall(\lambda, \mu) \in \mathcal{H} \times \mathcal{H}_{+},
\end{aligned}
$$

we can write, thanks to (2.5), the estimates

$$
\begin{gathered}
V_{p, r}^{\delta}\left(\lambda_{p, r}^{\delta, \alpha(\delta)}, \mu_{p, r}^{\delta, \alpha(\delta)}\right) \geq V_{p, r}^{0}(\lambda, \mu)+\alpha(\delta)\left\|\lambda_{p, r}^{\delta, \alpha(\delta)}\right\|^{2}+\alpha(\delta)\left\|\mu_{p, r}^{\delta, \alpha(\delta)}\right\|^{2}- \\
C \delta(1+\|\lambda\|+\|\mu\|)-\alpha(\delta)\|\lambda\|^{2}-\alpha(\delta)\|\mu\|^{2}
\end{gathered}
$$




$$
\begin{gathered}
V_{p, r}^{0}\left(\lambda_{p, r}^{\delta, \alpha(\delta)}, \mu_{p, r}^{\delta, \alpha(\delta)}\right)=V_{p, r}^{\delta}\left(\lambda_{p, r}^{\delta, \alpha(\delta)}, \mu_{p, r}^{\delta, \alpha(\delta)}\right)+\left[V_{p, r}^{0}\left(\lambda_{p, r}^{\delta, \alpha(\delta)}, \mu_{p, r}^{\delta, \alpha(\delta)}\right)-V_{p, r}^{\delta}\left(\lambda_{p, r}^{\delta, \alpha(\delta)}, \mu_{p, r}^{\delta, \alpha(\delta)}\right)\right] \geq \\
V_{p, r}^{\delta}\left(\lambda_{p, r}^{\delta, \alpha(\delta)}, \mu_{p, r}^{\delta, \alpha(\delta)}\right)-C \delta\left(1+\left\|\lambda_{p, r}^{\delta, \alpha(\delta)}\right\|+\left\|\mu_{p, r}^{\delta, \alpha(\delta)}\right\|\right),
\end{gathered}
$$

whence we obtain

$$
\begin{gathered}
V_{p, r}^{0}\left(\lambda_{p, r}^{\delta, \alpha(\delta)}, \mu_{p, r}^{\delta, \alpha(\delta)}\right) \geq V_{p, r}^{0}(\lambda, \mu)+\alpha(\delta)\left\|\lambda_{p, r}^{\delta, \alpha(\delta)}\right\|^{2}+\alpha(\delta)\left|\mu_{p, r}^{\delta, \alpha(\delta)}\right|^{2}-C \delta\left(1+\left\|\lambda_{p, r}^{\delta, \alpha(\delta)}\right\|+\left\|\mu_{p, r}^{\delta, \alpha(\delta)}\right\|\right)- \\
C \delta(1+\|\lambda\|+\|\mu\|) \quad \forall(\lambda, \mu) \in \mathcal{H} \times \mathcal{H}_{+} .
\end{gathered}
$$

From here, we deduce, due to the consistency condition (3.1) and limit relations (3.9), that for any fixed $M>0$ and for any fixed $\epsilon>0$ there exists such $\delta(\epsilon)>0$ for which the estimate

$$
\begin{aligned}
& V_{p, r}^{0}\left(\lambda_{p, r}^{\delta, \alpha(\delta)}, \mu_{p, r}^{\delta, \alpha(\delta)}\right) \geq \sup _{(\lambda, \mu) \in \mathcal{H} \times \mathcal{H}_{+}:\|\lambda\| \leq M,\|\mu\| \leq M} V_{p, r}^{0}(\lambda, \mu)-\epsilon \\
& \forall \delta \leq \delta(\epsilon) \forall(\lambda, \mu) \in\left\{(\lambda, \mu) \in \mathcal{H} \times \mathcal{H}_{+}:\|\lambda\| \leq M,\|\mu\| \leq M\right\}
\end{aligned}
$$

holds.

Suppose now that the limit relation (3.11) is not true. Then there exists such a sequence $\delta_{s}, s=1,2, \ldots$ convergent to zero that the inequality

$$
V_{p, r}^{0}\left(\lambda_{p, r}^{\delta_{s}, \alpha\left(\delta_{s}\right)}, \mu_{p, r}^{\delta_{s}, \alpha\left(\delta_{s}\right)}\right) \leq \sup _{(\lambda, \mu) \in \mathcal{H} \times \mathcal{H}_{+}} V_{p, r}^{0}(\lambda, \mu)-l, \quad s=1,2, \ldots
$$

is fulfilled for some $l>0$.

Since

$$
\sup _{(\lambda, \mu) \in \mathcal{H} \times \mathcal{H}_{+}} V_{p, r}^{0}(\lambda, \mu)-\sup _{(\lambda, \mu) \in \mathcal{H} \times \mathcal{H}_{+}:\|\lambda\| \leq M,\|\mu\| \leq M} V_{p, r}^{0}(\lambda, \mu) \rightarrow 0,
$$

for $M \rightarrow+\infty$, we deduce from the last estimate that for all sufficiently large positive $M$ the inequality

$$
V_{p, r}^{0}\left(\lambda_{p, r}^{\delta_{s}, \alpha\left(\delta_{s}\right)}, \mu_{p, r}^{\delta_{s}, \alpha\left(\delta_{s}\right)}\right) \leq \sup _{(\lambda, \mu) \in \mathcal{H} \times \mathcal{H}_{+}:\|\lambda\| \leq M,\|\mu\| \leq M} V_{p, r}^{0}(\lambda, \mu)-l / 2
$$

is true. This estimate contradicts to the estimate obtained above (3.12). The last contradiction proves correctness of the limit relation (3.11).

Summarizing the above arguments, we assert that the following "convergence" theorem for the dual regularization method in Problem $\left(P_{p, r}^{0}\right)$ is valid.

Theorem 1. Let Problem $\left(P_{p, r}^{0}\right)$ be solvable. Regardless of the properties of the solvability of the dual problem to Problem $\left(P_{p, r}^{0}\right)$ or, in other words, regardless of the properties of the subdifferential $\partial \beta(p, r)$ (it is empty or not empty), it is true that exist elements $\pi^{\delta} \in U^{\delta}\left[\lambda_{p, r}^{\delta, \alpha(\delta)}, \mu_{p, r}^{\delta, \alpha(\delta)}\right]$ such that the relations

$$
\begin{gathered}
g_{0}^{0}\left(\pi^{\delta}\right) \rightarrow g_{0}^{0}\left(\pi_{p, r}^{0}\right), \quad g_{1}^{0}\left(\pi^{\delta}\right)-h^{0}-p \rightarrow 0, \quad g_{2}^{0}\left(\pi^{\delta}\right)-r \leq \kappa(\delta), \quad\|\kappa(\delta)\| \rightarrow 0, \quad \delta \rightarrow 0, \\
\left\langle\left(\lambda_{p, r}^{\delta, \alpha(\delta)}, \mu_{p, r}^{\delta, \alpha(\delta)}\right),\left(g_{1}^{\delta}\left(\pi^{\delta}\right)-h^{\delta}-p, g_{2}^{\delta}\left(\pi^{\delta}\right)-r\right)\right\rangle \rightarrow 0, \quad \delta \rightarrow 0
\end{gathered}
$$

hold, in which the inequality $g_{2}^{0}\left(\pi^{\delta}\right)-r \leq \kappa(\delta)$ is understood in the sense of ordering on a cone of nonpositive functions in $\mathcal{H}$. Simultaneously, the equality

$$
\lim _{\delta \rightarrow+0} V_{p, r}^{0}\left(\lambda_{p, r}^{\delta, \alpha(\delta)}, \mu_{p, r}^{\delta, \alpha(\delta)}\right)=\sup _{(\lambda, \mu) \in \mathcal{H} \times \mathcal{H}_{+}} V_{p, r}^{0}(\lambda, \mu)
$$

is valid. In addition, the duality relation (2.3) holds. If the dual of Problem $\left(P_{p, r}^{0}\right)$ is solvable, then the limit relation $\left(\lambda_{p, r}^{\delta, \alpha},(\delta), \mu_{p, r}^{\delta, \alpha}\right) \rightarrow\left(\lambda_{p, r}^{0}, \mu_{p, r}^{0}\right), \delta \rightarrow 0$ is valid also, where $\left(\lambda_{p, r}^{0}, \mu_{p, r}^{0}\right)$ denotes the minimum-norm solution of the dual problem. 


\subsection{Stable sequential Lagrange principle for optimal control problem with pointwise state constraints}

We formulate in this subsection the necessary and sufficient condition for existence of a minimizing approximate solution in Problem $\left(P_{p, r}^{0}\right)$. Also, for this problem it can be called by stable sequential Lagrange principle in nondifferential form. Simultaneously, as we deal only with regular Lagrange function, the formulated theorem may be called by Kuhn-Tucker theorem in nondifferential form. Note that the necessity of the conditions of the theorem formulated below follows from the theorem 1. At the same time, their sufficiency is a simple consequence of the convexity of Problem $\left(P_{p, r}^{0}\right)$ and the conditions on its input data. A verification of these propositions for similar situation of the convex programming problem in a Hilbert space may be found in $[10,15,16]$.

Theorem 2. Regardless of the properties of the subdifferential $\partial \beta(p, r)$ (it is empty or not empty) or, in other words, regardless of the properties of the solvability of the dual problem to Problem $\left(P_{p, r}^{0}\right)$, necessary and sufficient conditions for Problem $\left(P_{p, r}^{0}\right)$ to have a minimizing approximate solution is that there is a sequence of dual variables $\left(\lambda^{k}, \mu^{k}\right) \in \mathcal{H} \times \mathcal{H}_{+}, k=1,2, \ldots$, such that $\delta^{k}\left\|\left(\lambda^{k}, \mu^{k}\right)\right\| \rightarrow 0, k \rightarrow \infty$, and relations

$$
\begin{gathered}
\pi^{\delta^{k}}\left[\lambda^{k}, \mu^{k}\right] \in \mathcal{D}_{p, r}^{\delta^{k}, \epsilon^{k}}, \quad \epsilon^{k} \rightarrow 0, \quad k \rightarrow \infty, \\
\left\langle\left(\lambda^{k}, \mu^{k}\right),\left(g_{1}^{\delta^{k}}\left(\pi^{\delta^{k}}\left[\lambda^{k}, \mu^{k}\right]\right)-h^{\delta^{k}}-p, g_{2}^{\delta^{k}}\left(\pi^{\delta^{k}}\left[\lambda^{k}, \mu^{k}\right]\right)-r\right)\right\rangle \rightarrow 0, \quad k \rightarrow \infty
\end{gathered}
$$

hold for some elements $\pi^{\delta^{k}}\left[\lambda^{k}, \mu^{k}\right] \in U^{\delta^{k}}\left[\lambda^{k}, \mu^{k}\right]$. The sequence $\pi^{\delta^{k}}\left[\lambda^{k}, \mu^{k}\right], k=1,2, \ldots$, is the desired minimizing approximate solution and each of its weak limit points is a solution of Problem $\left(P_{p, r}^{0}\right) . \quad A s\left(\lambda^{k}, \mu^{k}\right) \in \mathcal{H} \times \mathcal{H}_{+}, k=1,2, \ldots$, we can use the sequence of the points $\left(\lambda_{p, r}^{\delta^{k}, \alpha\left(\delta^{k}\right)}, \mu_{p, r}^{\delta^{k}, \alpha\left(\delta^{k}\right)}\right), k=1,2, \ldots$, generated by the dual regularization method of the theorem 1. If the dual of Problem $\left(P_{p, r}^{0}\right)$ is solvable, the sequence $\left(\lambda^{k}, \mu^{k}\right) \in \mathcal{H} \times \mathcal{H}_{+}, k=1,2, \ldots$, should be assumed to be bounded. The limit relation

$$
V_{p, r}^{0}\left(\lambda^{k}, \mu^{k}\right) \rightarrow \sup _{(\lambda, \mu) \in \mathcal{H} \times \mathcal{H}_{+}} V_{p, r}^{0}(\lambda, \mu)
$$

holds as a consequence of the relations (3.14), (3.15). Furthermore, each weak limit point (if such points exist) of the sequence $\left(\lambda^{k}, \mu^{k}\right) \in \mathcal{H} \times \mathcal{H}_{+}, \quad k=1,2, \ldots$ is a solution of the dual problem $V_{p, r}^{0}(\lambda, \mu) \rightarrow \max , \quad(\lambda, \mu) \in \mathcal{H} \times \mathcal{H}_{+}$.

P r o o f. To prove the necessity, we first note that problem $\left(P_{p, r}^{0}\right)$ is solvable (i.e., $U_{p, r}^{0} \neq$ $\emptyset)$ due to the conditions on the initial data and to the existence of a minimizing approximate solution. Now the existence of the indicated sequence $\left(\lambda^{k}, \mu^{k}\right) \in \mathcal{H} \times \mathcal{H}_{+}, k=1,2, \ldots$ and the limit relations (3.14) and (3.15) follow from Theorem 1 if the points $\left(\lambda^{k}, \mu^{k}\right)$ and $\pi^{\delta^{k}}\left[\lambda^{k}, \mu^{k}\right]$ are defined as $\left(\lambda_{p, r}^{\delta^{k}, \alpha\left(\delta^{k}\right)}, \mu_{p, r}^{\delta^{k}, \alpha\left(\delta^{k}\right)}\right)$, and $\pi_{\delta^{k}}, k=1,2, \ldots$, respectively. These limit relations imply that (3.16) holds as well. Really, combining estimates (2.4) with the limit relation $\delta^{k}\left\|\left(\lambda^{k}, \mu^{k}\right)\right\| \rightarrow 0, k \rightarrow \infty$, we conclude (see the estimate (2.5)) that $V_{p, r}^{\delta^{k}}\left(\lambda^{k}, \mu^{k}\right)-V_{p, r}^{0}\left(\lambda^{k}, \mu^{k}\right) \rightarrow 0, k \rightarrow \infty$. Then, in view of $(2.3),(3.15)$, and the limit relation $f^{0}\left(z^{\delta^{k}}\left[\lambda^{k}, \mu^{k}\right]\right) \rightarrow f^{0}\left(z_{p, r}^{0}\right), \quad k \rightarrow \infty$ (see (3.13)), we have

$V_{p, r}^{\delta^{k}}\left(\lambda^{k}, \mu^{k}\right)=f^{\delta^{k}}\left(z^{\delta^{k}}\left[\lambda^{k}, \mu^{k}\right]\right)+\left\langle\left(\lambda^{k}, \mu^{k}\right),\left(A^{\delta^{k}} z^{\delta^{k}}\left[\lambda^{k}, \mu^{k}\right]-h^{\delta^{k}}-p, g^{\delta^{k}}\left(z^{\delta^{k}}\left[\lambda^{k}, \mu^{k}\right]\right)-r\right)\right\rangle \rightarrow f^{0}\left(z_{p, r}^{0}\right)$,

therefore, the limit relation (3.16) holds true.

To prove the sufficiency, we first note also that the set $U_{p, r}^{0} \subset \mathcal{D}_{p, r}^{0 \epsilon^{k}}$ is not empty. This follows from the inclusion $\pi^{\delta^{k}}\left[\lambda^{k}, \mu^{k}\right] \in \mathcal{D}_{p, r}^{\delta^{k} \epsilon^{k}}$, from the fact that the sequence $\pi^{\delta^{k}}\left[\lambda^{k}, \mu^{k}\right], k=1,2, \ldots$ 
is bounded, and from the conditions on the initial data in Problem $\left(P_{p, r}^{0}\right)$. Furthermore, since the point $\pi^{\delta^{k}}\left[\lambda^{k}, \mu^{k}\right]$ minimizes on $\mathcal{D}$ the functional $L_{p, r}^{\delta^{k}}\left(\cdot, \lambda^{k}, \mu^{k}\right)$, we have

$$
\begin{gathered}
g_{0}^{\delta^{k}}\left(\pi^{\delta^{k}}\left[\lambda^{k}, \mu^{k}\right]\right)+\left\langle\left(\lambda^{k}, \mu^{k}\right),\left(g_{1}^{\delta^{k}}\left(\pi^{\delta^{k}}\left[\lambda^{k}, \mu^{k}\right]\right)-h^{\delta^{k}}-p, g_{2}^{\delta^{k}}\left(\pi^{\delta^{k}}\left[\lambda^{k}, \mu^{k}\right]\right)-r\right)\right\rangle \leq \\
g_{0}^{\delta^{k}}(\pi)+\left\langle\left(\lambda^{k}, \mu^{k}\right),\left(g_{1}^{\delta^{k}}(\pi)-h^{\delta^{k}}-p, g_{2}^{\delta^{k}}(z)-r\right)\right\rangle \quad \forall \pi \in \mathcal{D} .
\end{gathered}
$$

By the assumptions of the theorem, it follows that

$g_{0}^{\delta^{k}}\left(\pi^{\delta^{k}}\left[\lambda^{k}, \mu^{k}\right]\right) \leq g_{0}^{\delta^{k}}(\pi)+\left\langle\left(\lambda^{k}, \mu^{k}\right),\left(g_{1}^{\delta^{k}}(\pi)-h^{\delta^{k}}-p, g_{2}^{\delta^{k}}(\pi)-r\right)\right\rangle+\psi^{k} \quad \forall \pi \in \mathcal{D}, \quad \psi^{k} \rightarrow 0, \quad k \rightarrow \infty$.

Setting $\pi=\pi_{p, r}^{0} \in U_{p, r}^{0}$ and using the consistency condition $\delta^{k}\left\|\left(\lambda^{k}, \mu^{k}\right)\right\| \rightarrow 0, k \rightarrow \infty$, we obtain $g_{0}^{0}\left(\pi^{\delta^{k}}\left[\lambda^{k}, \mu^{k}\right]\right) \leq g_{0}^{0}\left(\pi_{p, r}^{0}\right)+\widetilde{\psi}^{k}, \widetilde{\psi}^{k} \rightarrow 0, k \rightarrow \infty$. Since we also have the inclusion $\pi^{\delta^{k}}\left[\lambda^{k}, \mu^{k}\right] \in \mathcal{D}_{p, r}^{\delta^{k}, \epsilon^{k}}$, using the classical weak compactness properties of a bounded convex closed set and the weak lower semicontinuity of a continuous convex functional in a Hilbert space, we easily derive $g_{0}^{0}\left(\pi^{\delta^{k}}\left[\lambda^{k}, \mu^{k}\right]\right) \rightarrow g_{0}^{0}\left(\pi_{p, r}^{0}\right), k \rightarrow \infty$; i.e., the sequence $\pi^{\delta^{k}}\left[\lambda^{k}, \mu^{k}\right], k=1,2, \ldots$ is a minimizing approximate solution in Problem $\left(P_{p, r}^{0}\right)$. In view of (3.15) and the obtained limit relation $g_{0}^{0}\left(\pi^{\delta^{k}}\left[\lambda^{k}, \mu^{k}\right]\right) \rightarrow g_{0}^{0}\left(\pi_{p, r}^{0}\right), k \rightarrow \infty$, we can write

$V_{p, r}^{\delta^{k}}\left(\lambda^{k}, \mu^{k}\right)=g_{0}^{\delta^{k}}\left(\pi^{\delta^{k}}\left[\lambda^{k}, \mu^{k}\right]\right)+\left\langle\left(\lambda^{k}, \mu^{k}\right),\left(g_{1}^{\delta^{k}}\left(\pi^{\delta^{k}}\left[\lambda^{k}, \mu^{k}\right]\right)-h^{\delta^{k}}-p, g_{2}^{\delta^{k}}\left(\pi^{\delta^{k}}\left[\lambda^{k}, \mu^{k}\right]\right)-r\right)\right\rangle \rightarrow g_{0}^{0}\left(\pi_{p, r}^{0}\right)$,

therefore, limit relation (3.16) holds by virtue of estimate (2.5), equality (2.3), and the limit relation $\delta^{k}\left\|\left(\lambda^{k}, \mu^{k}\right)\right\| \rightarrow 0, k \rightarrow \infty$. To conclude, we note that, it is easy to show that each weak limit point of the sequence $\left(\lambda^{k}, \mu^{k}\right) \in \mathcal{H} \times \mathcal{H}_{+}, k=1,2, \ldots$ (if such points exist) is a solution of the dual problem $V_{p, r}^{0}(\lambda, \mu) \rightarrow \max , \quad(\lambda, \mu) \in \mathcal{H} \times \mathcal{H}_{+}$.

Remark 1. If the functional $g_{0}^{0}$ is strongly convex and subdifferentiable on $\mathcal{D}$ then from the weak convergence of the unique in this case elements $\pi^{\delta^{k}}\left[\lambda^{k}, \mu^{k}\right]$ to unique element $\pi_{p, r}^{0}$ as $k \rightarrow$ $\infty$, and numerical convergence $g_{0}^{0}\left(\pi^{\delta^{k}}\left[\lambda^{k}, \mu^{k}\right]\right) \rightarrow g_{0}^{0}\left(\pi_{p, r}^{0}\right), k \rightarrow \infty$ follows the strong convergence $\pi^{\delta^{k}}\left[\lambda^{k}, \mu^{k}\right] \rightarrow \pi_{p, r}^{0}, k \rightarrow \infty$. Problem $\left(P_{p, r}^{0}\right)$ with the strongly convex $g_{0}^{0}$ for linear system of ordinary differential equations but with exact input data is studied in [17].

\subsection{Stable sequential Pontryagin maximum principle for optimal control problem with pointwise state constraints}

Denote by $U_{\max }^{\delta}[\lambda, \mu]$ a set of elements $\pi_{\max }^{\delta}[\lambda, \mu] \in \mathcal{D}$ that satisfy all relations of the maximum principle (2.2) of the lemma 1. Under the supplementary condition of existence of continuous with respect to $z$ gradients $\nabla_{z} \varphi_{2}^{\delta}(x, t, z), \nabla_{z} G^{\delta}(x, z)$ with corresponding estimates, it follows that the proposition of the Theorem 2 may be rewritten in the form of the stable sequential Pontryagin maximum principle. It is obviously that the equality $U_{\max }^{\delta}[\lambda, \mu]=U^{\delta}[\lambda, \mu]$ takes place under mentioned supplementary condition.

Theorem 3. Regardless of the properties of the subdifferential $\partial \beta(p, r)$ (it is empty or not empty) or, in other words, regardless of the properties of the solvability of the dual problem to Problem $\left(P_{p, r}^{0}\right)$, necessary and sufficient conditions for Problem $\left(P_{p, r}^{0}\right)$ to have a minimizing approximate solution is that there is a sequence of dual variables $\left(\lambda^{k}, \mu^{k}\right) \in \mathcal{H} \times \mathcal{H}_{+}, k=1,2, \ldots$, such that $\delta^{k}\left\|\left(\lambda^{k}, \mu^{k}\right)\right\| \rightarrow 0, k \rightarrow \infty$, and relations (3.14), (3.15) hold for some elements $\pi^{\delta^{k}}\left[\lambda^{k}, \mu^{k}\right] \in$ $U_{\text {max }}^{\delta^{k}}\left[\lambda^{k}, \mu^{k}\right]$. Moreover, the sequence $\pi^{\delta^{k}}\left[\lambda^{k}, \mu^{k}\right], k=1,2, \ldots$, is the desired minimizing approximate solution and each of its weak limit points is a solution of Problem $\left(P_{p, r}^{0}\right) . A s\left(\lambda^{k}, \mu^{k}\right) \in \mathcal{H} \times \mathcal{H}_{+}$, 
$k=1,2, \ldots$, we can use the sequence of the points $\left(\lambda_{p, r}^{\delta^{k}, \alpha\left(\delta^{k}\right)}, \mu_{p, r}^{\delta^{k}, \alpha\left(\delta^{k}\right)}\right), k=1,2, \ldots$, generated by the dual regularization method of the theorem 1. If the dual of Problem $\left(P_{p, r}^{0}\right)$ is solvable, the sequence $\left(\lambda^{k}, \mu^{k}\right) \in \mathcal{H} \times \mathcal{H}_{+}, k=1,2, \ldots$, should be assumed to be bounded. The limit relation (3.16) holds as a consequence of the relations (3.14), (3.15).

Remark 2. When the inequality constraint in Problem $\left(P_{p, r}^{0}\right)$ is absent, i.e., $\left(P_{p, r}^{0}\right)=\left(P_{p}^{0}\right)$, and $\phi_{1}(x, t) \equiv 1$, the target functional $g_{0}^{0}$ is taken, for example, in the form $g_{0}^{0}(\pi) \equiv\|\pi\|^{2} \equiv\|u\|^{2}+\|w\|^{2}$ then Problem $\left(P_{p}^{0}\right)$ acquires the typical form of unstable inverse problem. In this case the stable sequential Pontryagin maximum principle of the Theorem 3 becomes a tool for the direct solving such unstable inverse problem.

Remark 3. In important partial case of Problem $\left(P_{p, r}^{0}\right)=\left(P_{r}^{0}\right)$, when it has only the inequality constraint $\left(\varphi_{1}^{\delta}(x, t)=h^{\delta}(x, t)=p(x, t)=0,(x, t) \in Q\right)$, "weak" passage to the limit in the relations of the Theorem 3 leads to usual for similar optimal control problems Pontryagin maximum principle (see, e.g., $[3,8])$ with nonnegative Radon measures in the input data of the adjoint equation.

\section{REFERENCES}

1. Alekseev V.M., Tikhomirov V.M., Fomin S.V. Optimal Control. Moscow: Nauka, 1979. 432 p. [in Russian]

2. Aubin J.-P., Ekeland I. Applied Nonlinear Analysis. New York: Wiley, 1984. 518 p.

3. Casas E., Raymond J.-P., Zidani H. Pontryagin's principle for local solutions of control problems with mixed control-state constraints // SIAM J. Control Optim. 2000. Vol. 39, no. 4. P. 1182-1203.

4. Kalinin A.V., Sumin M.I., Tyukhtina A.A. Stable sequential Lagrange principles in the inverse final observation problem for the system of Maxwell equations in the quasistationary magnetic approximation // Differential Equations. 2016. Vol. 52, no. 5. P. 587-603.

5. Kuzenkov O.A., Plotnikov V.I. Existence and uniqueness of a generalized solution to a linear vector equation of parabolic type in the third boundary value problem // Mathematical Modeling and Optimization Methods (Gorky State University). 1989. P. 132-144. [in Russian].

6. Ladyzhenskaya O.A., Solonnikov V.A., Ural'tseva N.N. Linear and quasilinear equations of parabolic type. Providence, R.I.: Am. Math. Soc., 1968. 648 p.

7. Plotnikov V.I. Existence and uniqueness theorems and a priori properties of weak solutions // Dokl. Akad. Nauk SSSR. 1965. Vol. 165, no. 1. 33-35. [in Russian]

8. Raymond J.-P., Zidani H. Pontryagin's principle for state-constrained control problems governed by parabolic equations with unbounded controls // SIAM J. Control Optim. 1998. Vol. 36. no. 6. P. 18531879.

9. Gaikovich K.P., Gaikovich P.K., Sumin M.I. Stable sequential Kuhn-Tucker theorem in onedimensional inverse problems of dielectric reflectometry // Proc. of the 16th International Conference on Transparent Optical Networks: ICTON-2014. 2014. P. Th.A4.6. P. 1-4.

10. Sumin M.I. Stable sequential convex programming in a Hilbert space and its application for solving unstable problems // Comput. Math. Math. Phys. 2014. Vol. 54, no. 1. P. 22-44.

11. Sumin M.I. A regularized gradient dual method for the inverse problem of a final observation for a parabolic equation // Comput. Math. Math. Phys. 2004. Vol. 44, no. 11. P. 1903-1921.

12. Sumin M.I. Duality-based regularization in a linear convex mathematical programming problem // Comput. Math. Math. Phys. 2007. Vol. 47, no. 4. P. 579-600.

13. Sumin M.I. Regularized parametric Kuhn-Tucker theorem in a Hilbert space // Comput. Math. Math. Phys. 2011. Vol. 51, no. 9. P. 1489-1509.

14. Sumin M.I. Dual regularization and Pontryagin's maximum principle in a problem of optimal boundary control for a parabolic equation with nondifferentiable functionals // Proc. Steklov Inst. Math. 2011. Suppl. 1. P. S161-S177.

15. Sumin M.I. On the stable sequential Kuhn-Tucker theorem and its applications // Appl. Math. 2012. Vol. 3, no. 10A. P. 1334-1350. 
16. Sumin M.I. On the stable sequential Lagrange principle in the convex programming and its applications for solving unstable problems // Proc. of the Inst. of Math. and Mech., Ural Branch of the RAS. 2013. Vol. 19, no. 4. P. 231-240. [in Russian]

17. Sumin M.I. Parametric dual regularization for an optimal control problem with pointwise state constraints // Comput. Math. Math. Phys. 2009. Vol. 49, no. 12. P. 1987-2005.

18. Sumin M.I. Nekorrektnye zadachi i metody ikh resheniya. Materialy k lektsiyam dlya studentov starshikh kursov (Ill-Posed Problems and Solution Methods). Nizhnii Novgorod: Nizhnii Novgorod State University, 2009. 289 p. [in Russian]

19. Sumin M.I. Stable sequential Pontryagin maximum principle in optimal control problem with state constraints // Proc. of the XIIth All-Russia Conference on Control Problems, Inst. of Control Sci. of RAS, Moscow. 2014. P. 796-808. [in Russian]

20. Sumin M.I. Stable sequential Pontryagin maximum principle in optimal control for distributed systems // Proc. of Intern. conf. "Systems Dynamics and Control Processes" dedicated to the 90-th anniversary of academician N.N. Krasovskii (Ekaterinburg, Russia, Sept. 15-20, 2014). Ekaterinburg: Ural Federal University, 2015. P. 301-308. [in Russian]

21. Sumin M.I. Subdifferentiability of value functions and regularization of Pontryagin maximum principle in optimal control for distributed systems / / Tambov State University Reports. Series: Natural and Tech. Sci. 2015. Vol. 20, no. 5. P. 1461-1477. [in Russian]

22. Sumin M.I. The first variation and Pontryagin's maximum principle in optimal control for partial differential equations // Comput. Math. Math. Phys. 2009. Vol. 49, no. 6. P. 958-978.

23. Warga J. Optimal control of differential and functional equations. New York: Academic Press, 1972. $531 \mathrm{p}$. 